\title{
Evaluation of the clinical efficacy of preserving the left colic artery in laparoscopic resection for rectal cancer: A meta-analysis
}

\author{
DONGWEI FAN ${ }^{*}$, CHENSONG ZHANG ${ }^{*}$, XUANHE LI, CHANGYANG YAO and TINGJING YAO \\ Department of Surgical Oncology, The First Affiliated Hospital of Bengbu Medical College, Bengbu, Anhui 233000, P.R. China
}

Received February 14, 2018; Accepted August 31, 2018

DOI: $10.3892 / \mathrm{mco} .2018 .1714$

\begin{abstract}
The aim of the present meta-analysis compared left colic artery (LCA) preservation with non-preservation in laparoscopic resection of rectal cancer in terms of feasibility, efficacy and safety. The PubMed, Ovid, Embase, Web of Science, CBM, CNKI, VIP and WanFang Data databases were searched prior to June 2017 for studies comparing LCA preservation and non-preservation in laparoscopic resection for rectal cancer. Two researchers screened the literature independently, extracted the data and evaluated the risk of bias. The study was performed using RevMan 5.3 software for meta-analysis. A total of 10 studies comparing LCA preservation and non-preservation in laparoscopic resection for rectal cancer were selected for this meta-analysis, with a combined study population of 1,471 patients. The results of the meta-analysis demonstrated that, when comparing LCA preservation with non-preservation in laparoscopic resection for rectal cancer, there were significant differences between the two groups in terms of operative time $(\mathrm{P}<0.01)$, estimated blood loss $(\mathrm{P}<0.01)$, percentage of neostomy $(\mathrm{P}<0.01)$, the number of retrieved lymph nodes $(\mathrm{P}<0.01)$, time to first postoperative exhaust $(\mathrm{P}<0.01)$ and amount of anastomotic leakage $(\mathrm{P}<0.01)$. However, there were no significant differences in postoperative hospital stay $(\mathrm{P}=0.28)$, incidence of recurrence $(\mathrm{P}=0.73)$ and incidence of metastasis $(\mathrm{P}=0.52)$. Therefore, compared with LCA non-preservation, patients in whom the LCA was preserved during laparoscopic resection for rectal cancer had a better prognosis. However, there was no difference in recurrence or metastasis between the two groups. Although the operative time and estimated blood loss were increased with LCA preservation, these may be reduced with improving proficiency of the operating surgeons. The
\end{abstract}

Correspondence to: Dr Tingjing Yao, Department of Surgical Oncology, The First Affiliated Hospital of Bengbu Medical College, 287 Zhihuai Road, Bengbu, Anhui 233000, P.R. China

E-mail: 13855200468@163.com

${ }^{*}$ Contributed equally

Key words: left colic artery, laparoscopic, rectal cancer, meta-analysis conclusions of the present study require verification by larger samples and high-quality randomized controlled trials.

\section{Introduction}

Rectal cancer has the fourth highest incidence among malignant tumors in China, and $70 \%$ of rectal cancers are located in the lower rectum (1). Rectal cancer has a high mortality rate, mainly attributed to local recurrence, lymphatic metastasis and hematogenous metastasis (2). Surgical resection is the main treatment for rectal cancer. In recent years, with the rapid development of endoscopic techniques, laparoscopic resection of rectal cancer has become the preferred surgical method, as it involves less trauma, less pain, faster recovery, minor scarring and fewer complications, among other advantages (3). Total mesorectal excision (TME) is generally well-received by surgeons, although whether to preserve the left colic artery (LCA) during surgery remains controversial (4).

Preservation of the LCA involves the ligation of blood vessels in the lower part of the LCA that originates from the lower part of inferior mesenteric artery (IMA), while cutting the LCA involves the ligation of blood vessels near the origin of the IMA and dissection of the regional lymph nodes (5). However, further study is required to determine whether the effects of two surgical methods on patient prognosis differ significantly and which method is more beneficial for patients. To this end, systematic evaluation and meta-analysis were used to comprehensively evaluate the clinical efficacy of laparoscopic resection of rectal cancer with and without preservation of the LCA, hoping to provide information that may serve as a reference for decision making in clinical practice.

\section{Materials and methods}

Search strategy. Under the supervision of TJY and CYY, CSZ and DWF systematically searched the PubMed, Ovid, Embase, Web of Science, CBM, CNKI, VIP and WanFang Data databases prior to June 2017 for studies comparing LCA preservation and non-preservation in laparoscopic resection for rectal cancer. In addition, the reference lists of the studies were obtained to supplement the literature. The following free-text terms and MeSH terms were used to identify the studies: Left colic artery, left colonic artery, rectal cancer, rectal carcinoma, laparoscopic, randomized controlled trial and controlled clinical trial. 
Study selection and inclusion criteria. The inclusion criteria were as follows: i) Randomized controlled trial (RCT) or quasi-RCT, which is a quasi-randomised trial that uses a quasi-random method of allocating participants to different interventions (this design is frequently used when it is not logistically feasible or ethical to conduct an RCT); ii) patients with rectal cancer diagnosis without limitations regarding age, race, nationality or disease course; iii) laparoscopic resection performed for rectal cancer, with the experimental group retaining and the control group not retaining the LCA; and iv) the results referred to at least one quantitative study. The data extraction included: i) Basic information about the research, including title, author, publication date, etc.; ii) the baseline objective and the details of the intervention; iii) key elements of the risk assessment; and iv) outcome indicators and outcome measurement data, including operative time, estimated blood loss, percentage of neostomy, amount of anastomotic leakage, number of retrieved IMA lymph nodes, postoperative hospital stay, incidence of recurrence and incidence of metastasis. The exclusion criteria were as follows: i) Previously published literature; ii) literature not published in Chinese or English; iii) loss of $>20 \%$ of patients during follow-up; iv) inability to extract the relevant data from the original literature or contact the author; and v) presence of tumors outside the rectum including extra-rectal metastasis and primary tumors associated with rectal cancer.

Quality assessment. Two researchers (CSZ and XHL) independently reviewed and extracted the required data, which were cross-checked. Disagreements were resolved by a third researcher ( $\mathrm{YW}$ ). Attempts were made to contact the original authors to supplement missing data. During literature screening, the title of the article we first read to exclude studies that were clearly irrelevant, and then the abstract and the full text were read to determine whether or not to include the study in question. The bias risk assessment included in the study was independently evaluated by two researchers (CSZ and CYY) based on the Cochrane Collaboration Network for RCT bias assessment tools to assess the risk of inclusion bias. Risk and bias were assessed in the following areas: Random sequence generation, allocation concealment, blinding of participants and personnel, blindness of outcome assessment, incomplete outcome date, selective reporting and other bias. The studies were divided into three groups based on the assessment of bias, namely high, low and unclear risk of bias. In the course of the assessment, in case of divergence, it was settled by discussion or submitted to a third researcher.

Statistical analysis. A total of 10 studies (6-15) involving 1,471 patients with rectal cancer were finally included in the meta-analysis (Fig. 1). The search strategy is summarized in File S1. Meta-analysis was performed using RevMan 5.3 software (Cochrane Community). Relative risk (RR) was calculated for enumeration data as effect indicators, and mean deviation (MD) was calculated for measurement data as effect indicators. Additionally, each effect indicator was given a point estimate and $95 \%$ confidence interval (CI) (16). The heterogeneity of the included studies was analyzed by $\chi^{2}$ test (test level $\alpha=0.1$ ) and quantitatively determined by $\mathrm{I}^{2}$ statistics. If there was no statistical heterogeneity among the results, the fixed-effects model was used for meta-analysis; if statistical heterogeneity was indeed present in the results of the study, the random-effects model was used for meta-analysis after the obvious clinical heterogeneity of the impact was excluded. Subgroup analysis or sensitivity analysis was used when clinical studies had significant heterogeneity or when only conducting descriptive analysis (17). The level of significance was $\alpha=0.05$.

\section{Results}

The literature screening process and results are presented in Fig. 1. The clinical characteristics and related information regarding the patients included in this study are shown in Table I. The main results of this study are as follows:

Operative time. A total of 9 RCTs (6,7,9-15) were included, involving 1,448 patients with rectal cancer. A meta-analysis, which applied the fixed-effects model, revealed that there was a significant difference between preservation and non-preservation of the LCA during laparoscopic resection of rectal cancer $(\mathrm{RR}=5.87$; 95\% CI: 2.60, 9.14; $\mathrm{P}<0.01$; Fig. 2), with the operative time for preservation of the LCA being comparatively longer.

Estimated blood loss. A total of 9 RCTs (7-9,11-15) were included, involving 1,346 patients with rectal cancer. A meta-analysis, which applied the fixed-effects model, revealed that there was a significant difference between preservation and non-preservation of the LCA during laparoscopic resection of rectal cancer $(\mathrm{RR}=3.92 ; 95 \% \mathrm{CI} ; 2.04,5.81 ; \mathrm{P}<0.01$; Fig. 3). In a comparison of the two groups, preserving the LCA was associated with a larger volume of blood loss.

Number of retrieved lymph nodes. A total of 5 RCTs $(7,9-11,15)$ were included, involving 961 patients with rectal cancer. A meta-analysis, which applied the fixed-effects model, revealed that there was a significant difference between preservation and non-preservation of the LCA in laparoscopic resection of rectal cancer ( $R R=-2.28$; 95\% CI: -3.08, -1.48; P<0.01; Fig. 4), higher number of retrieved nodes were swept with LCA preservation.

Time to first postoperative exhaust. A total of 4 RCTs $(6,7,13,14)$ were included, involving 207 patients with rectal cancer. A meta-analysis, which applied the fixed-effects model, revealed that there was a significant difference between preservation and non-preservation of the LCA in laparoscopic resection of rectal cancer $(\mathrm{RR}=-0.46$; 95\% CI: $-0.60,-0.31$; $\mathrm{P}<0.01$; Fig. 5$)$, as the time to the first postoperative exhaust was reduced in patients in whom the LCA was preserved.

Postoperative hospital stay. A total of 4 RCTs $(7,9,10,15)$ were included, involving 912 patients with rectal cancer. A meta-analysis, which applied the fixed-effects model, revealed that there was no statistically significant difference in postoperative hospital stay between preservation and non-preservation of the LCA in laparoscopic resection of rectal cancer $(\mathrm{RR}=-0.29$; 95\% CI: $-0.81,0.23$; $\mathrm{P}=0.28$; Fig. 6).

Neostomy. A total of $4 \operatorname{RCTs}(6,10,13,14)$ were included, involving 266 patients with rectal cancer. A meta-analysis, 


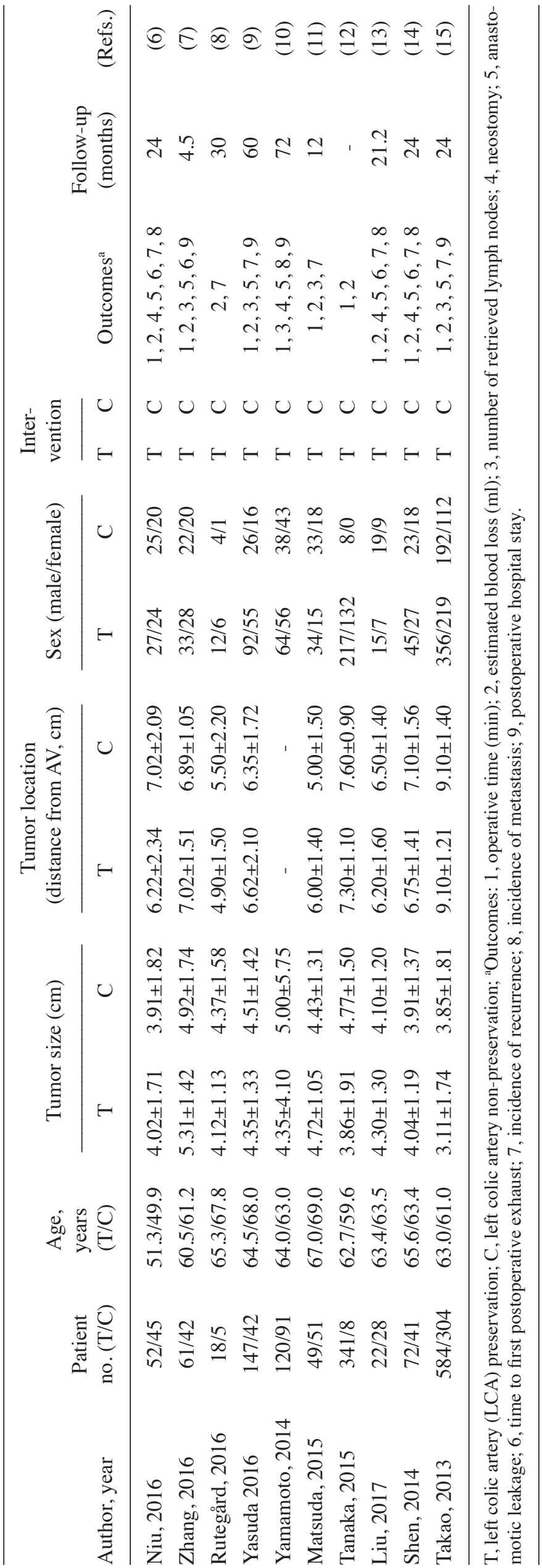




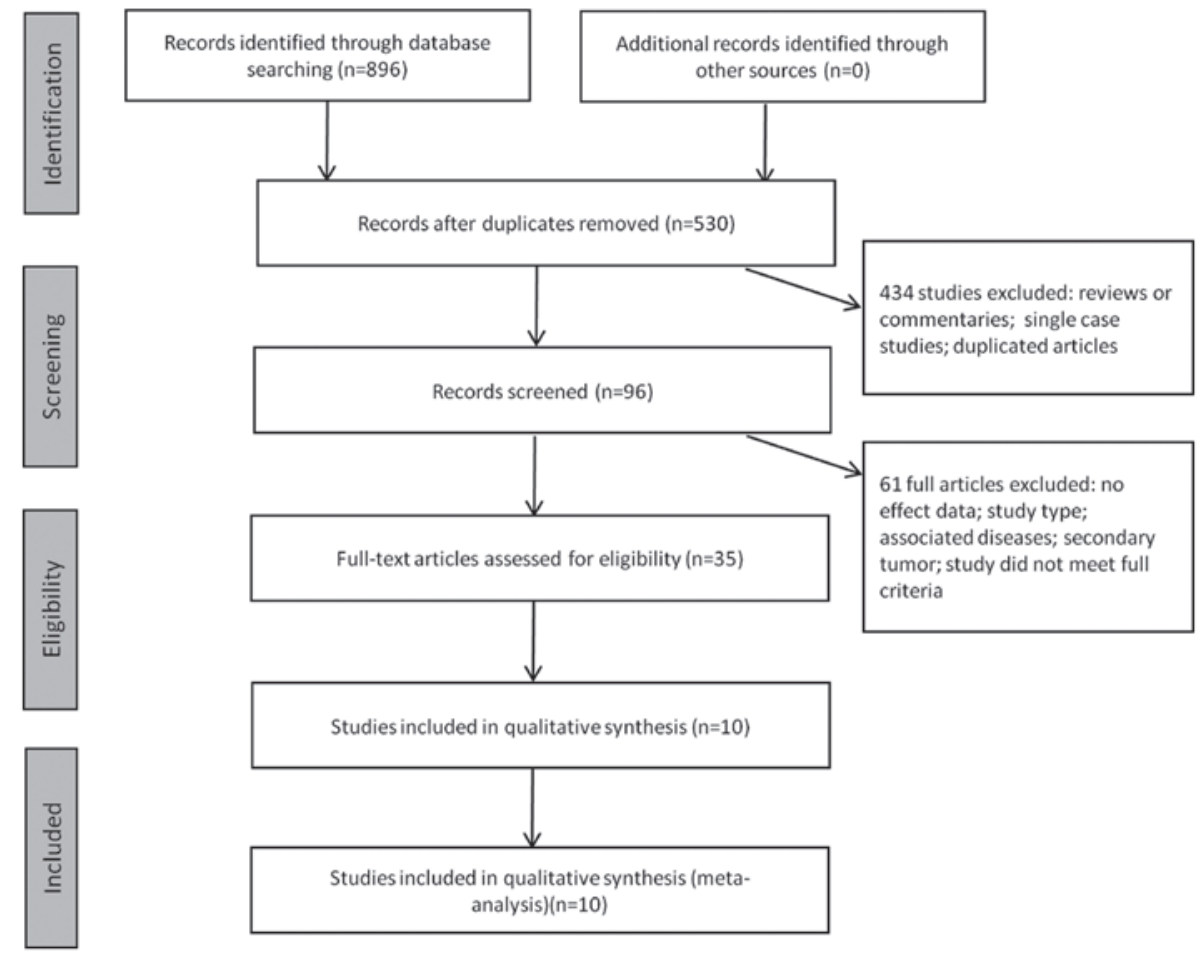

Figure 1. Flow chart of the literature search and article selection.

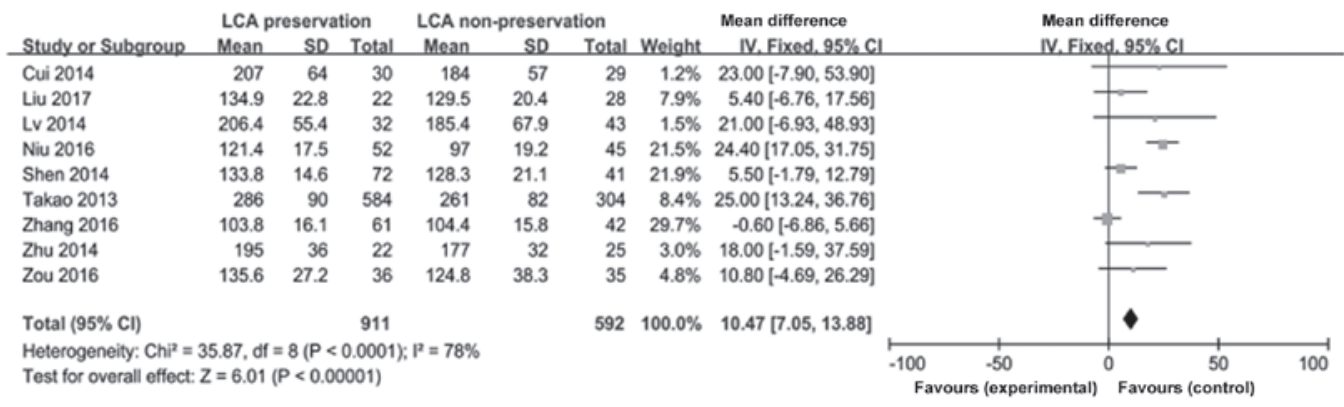

Figure 2. Meta-analysis of operative time between the two groups.

which applied the fixed-effects model, revealed that there was a significant difference between preservation and non-preservation of the LCA in laparoscopic resection of rectal cancer $(\mathrm{RR}=0.16,95 \% \mathrm{CI}: 0.04,0.62 ; \mathrm{P}=0.008$; Fig. 7), with the patients in whom the LCA was preserved having a lower percentage of neostomy.

Anastomotic leakage. A total of 7 RCTs (6,7,9,10,13-15) were included, involving 1,058 patients with rectal cancer. A meta-analysis, which applied the fixed-effects model, revealed that there was a significant difference between preservation and non-preservation of the LCA in laparoscopic resection of rectal cancer $(\mathrm{RR}=0.48,95 \% \mathrm{CI}$ : 0.32, 0.71; $\mathrm{P}<0.01$; Fig. 8), with patients in whom the LCA was preserved being less likely to experience anastomotic leakage.

Recurrence. A total of 7 RCTs $(6,8,9,11,13-15)$ were included, involving 944 patients with rectal cancer. A meta-analysis, which applied the fixed-effects model, revealed that there was no statistically significant difference in the risk of recurrence between preservation and non-preservation of the LCA in laparoscopic resection of rectal cancer $(\mathrm{RR}=0.93$; $95 \% \mathrm{CI}$ : 0.60, 1.44; P=0.73; Fig. 9).

Metastasis. A total of 4 RCTs $(6,10,13,14)$ were included, involving 266 patients with rectal cancer. A meta-analysis, which applied the fixed-effects model, revealed that there was no statistically significant difference in the risk of metastasis between preservation and non-preservation of the LCA in laparoscopic resection of rectal cancer $(\mathrm{RR}=0.81 ; 95 \% \mathrm{CI}$ : $0.43,1.53$; $\mathrm{P}=0.52$; Fig. 10).

Risk of bias assessment. Two researchers (CSZ and XHL) independently assessed the risks and bias of the included studies. The contents of the specific assessments included were as follows: Selection bias, performance bias, attrition bias, reporting bias and other types of bias (18). The details of the Cochrane risk of bias assessment are shown in Fig. 11. 


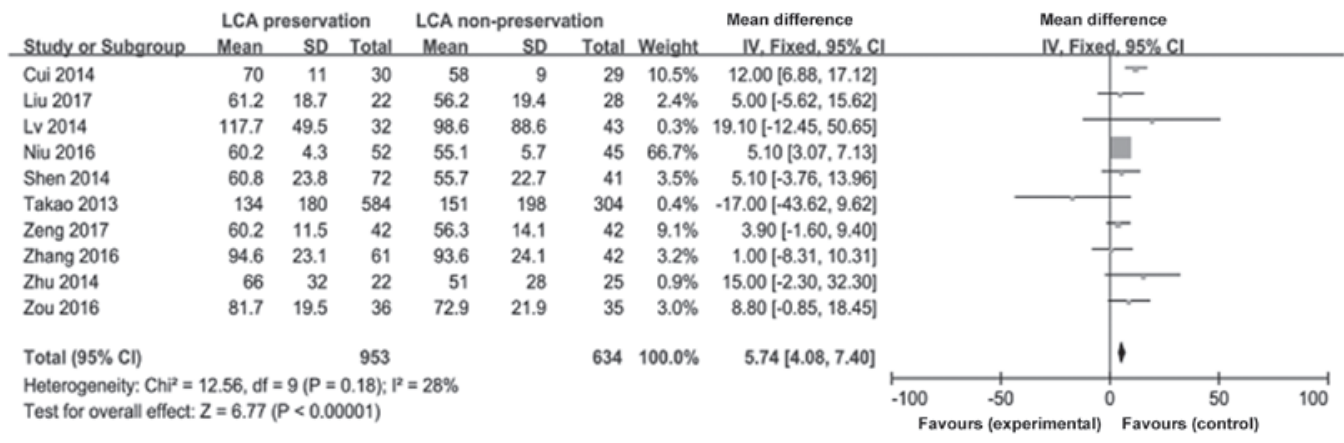

Figure 3. Meta-analysis of intraoperative blood loss between the two groups.

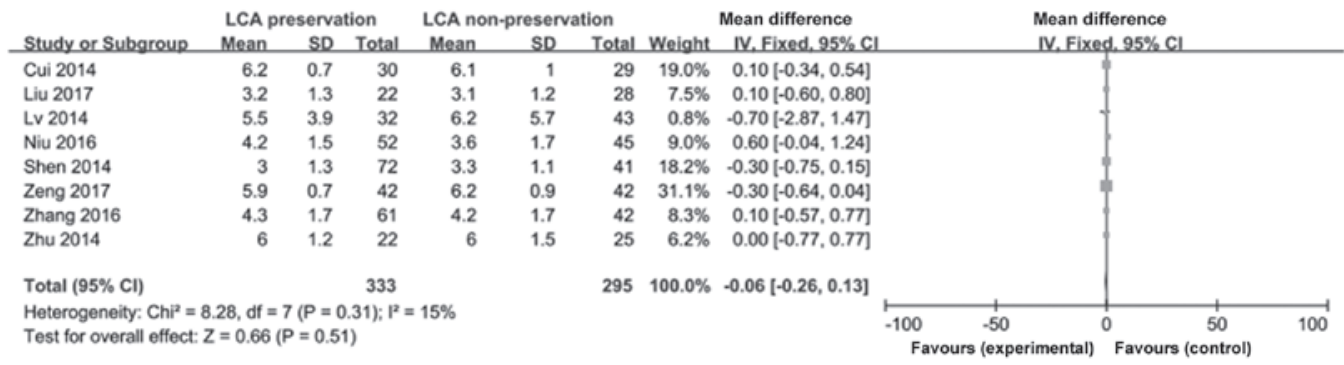

Figure 4. Meta-analysis of the number of retrieved lymph nodes between the two groups.

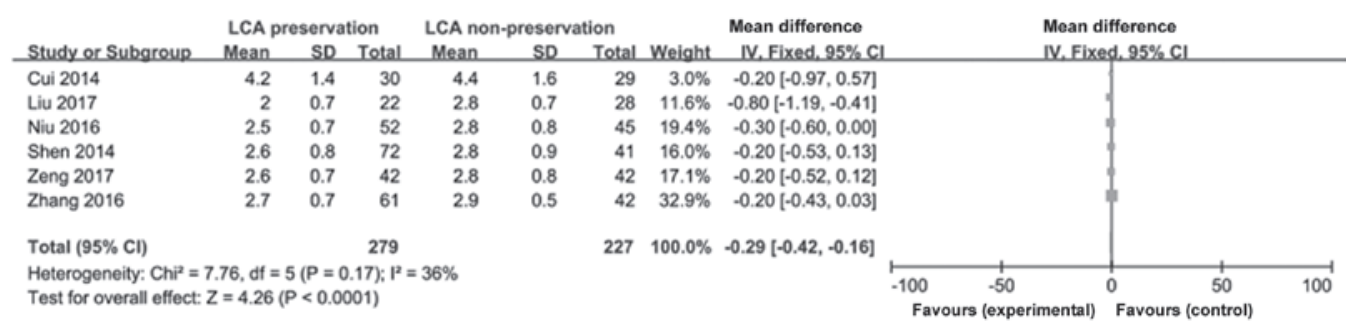

Figure 5. Meta-analysis of time to first postoperative exhaust between the two groups.

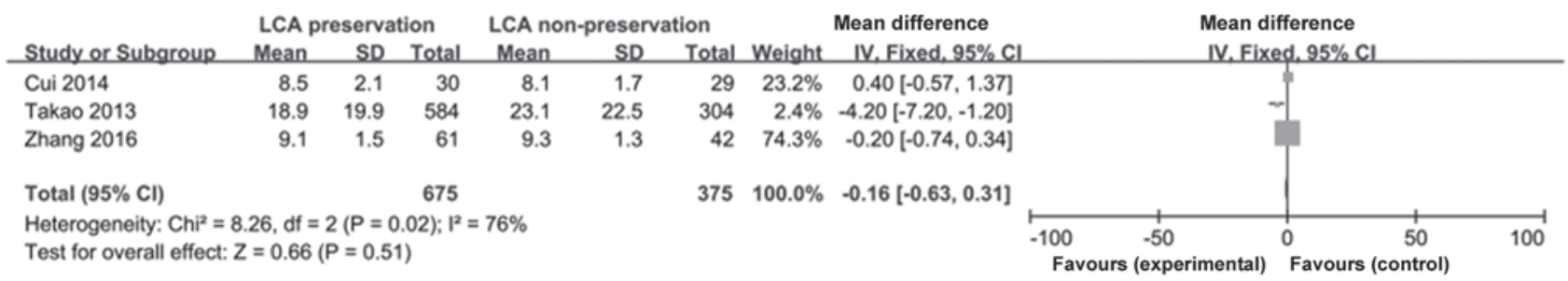

Figure 6. Meta-analysis of postoperative hospital stay between the two groups.

\section{Discussion}

The most serious complication of laparoscopic surgery for rectal cancer is anastomotic leakage, which leads to peritoneal infection and peritonitis, increasing the likelihood of a second surgery, prolonging hospital stay, and affecting patients undergoing chemotherapy and radiotherapy, which may pose a serious threat to the patients' life and well-being $(19,20)$. The principal causes of anastomotic leakage are diverse, such as anastomotic tension, compromised blood supply, hypoproteinemia, bleeding and blood transfusion and prolonged operative time. However, studies have demonstrated that the most frequent cause is anastomotic bowel blood supply disturbances $(21,22)$.

Traditional rectal cancer resection does not preserve the LCA due to high ligation of the IMA, and the anastomotic blood supply mainly comes from the marginal branch of the middle artery of the colon. Retention of the LCA may provide a better 


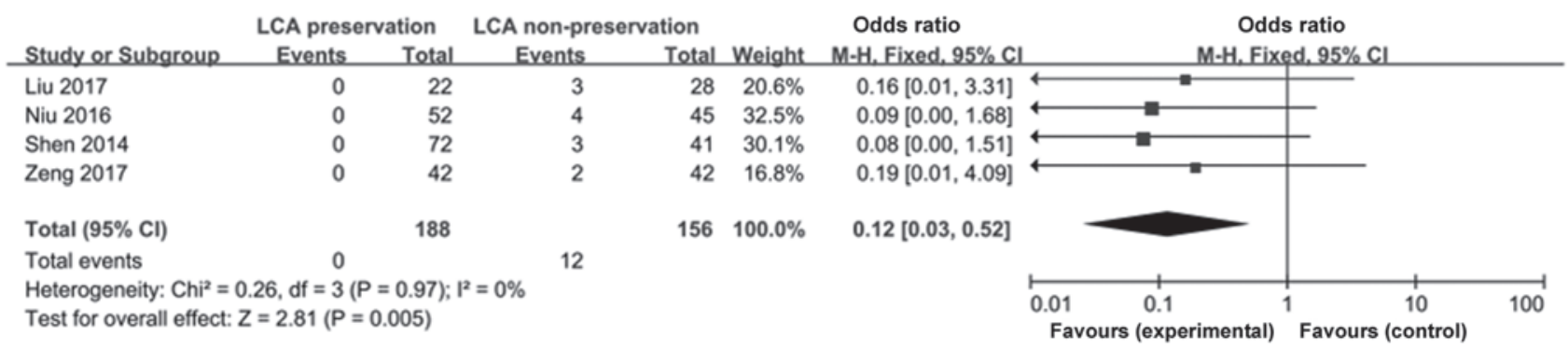

Figure 7. Meta-analysis of neostomy between the two groups.

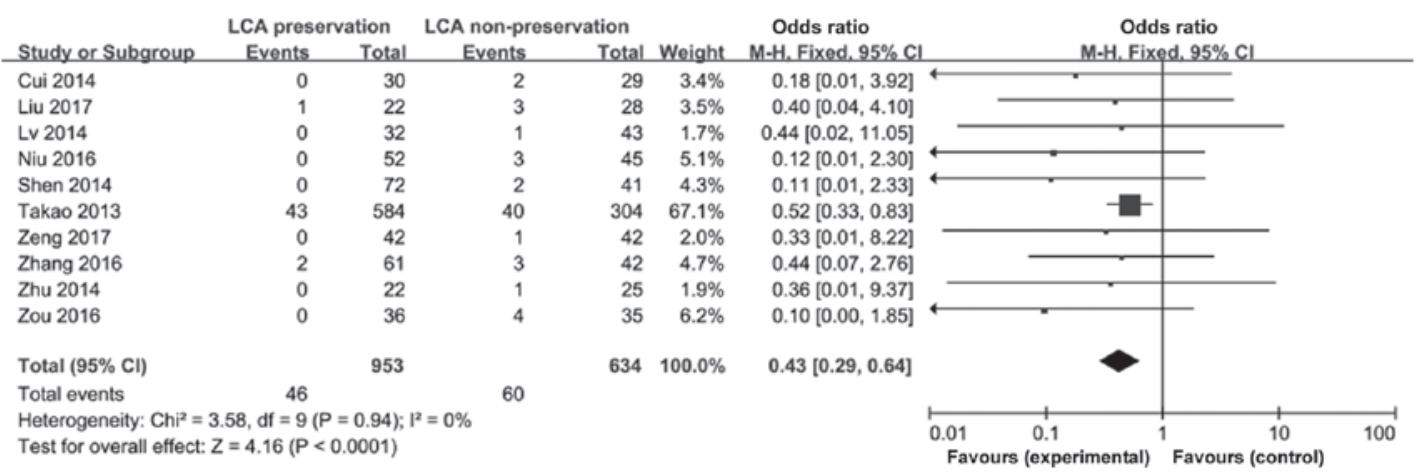

Figure 8. Meta-analysis of anastomotic leakage between the two groups.

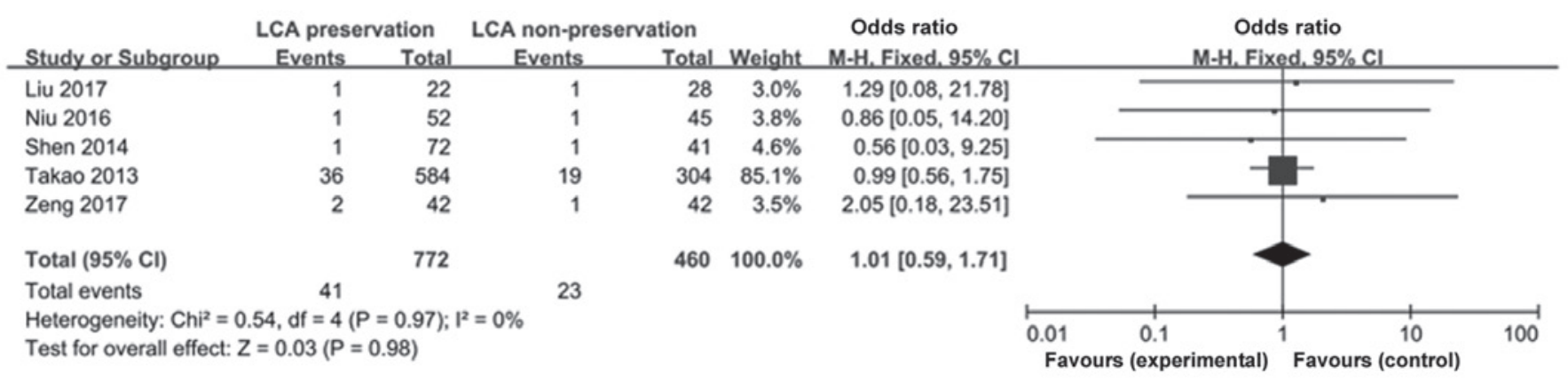

Figure 9. Meta-analysis of recurrence between the two groups.

blood supply for the proximal colon stump. This theory has been confirmed by intraoperative vascular Doppler surgery in clinical studies (23-25). Komen et al (26) also confirmed that the blood supply to the colon stump after preservation of the LCA was significantly better compared with non-preservation. However, high ligation of the IMA has more advantages in terms of lymph node dissection, as the nodes in the region of the mesenteric vascular root are more thoroughly dissected.

In the present study, systematic review and meta-analysis were used to evaluate the correlation between preservation and non-preservation of the LCA in laparoscopic resection for rectal cancer. The patient complications were systematically evaluated. Regarding intraoperative factors, operative time, intraoperative blood loss, lymph node dissection and the necessity of preventative colostomy were evaluated. In terms of postoperative complications, first time passing wind following operation, length of hospital stay, postoperative anastomotic leakage, recurrence and metastasis were evaluated. The results of the meta-analysis revealed that, compared with non-preservation, preservation of the LCA was associated with increased operative time and intraoperative blood loss. However, preserving the LCA was associated with faster recovery and lower incidence of anastomotic leakage. Additionally, higher surgeon proficiency may also reduce operative time and the volume of intraoperative blood loss. In this case, preservation of the LCA in patients with rectal cancer may improve the prognosis.

The limitations of this study include the following: i) The number of studies and the total number of cases is relatively small, and the meta-analysis of test performance may still be insufficient; ii) part of the studies do not describe the method for generation of random sequence and the allocation of hidden methods, which may indicate the presence of selective bias and implementation bias; iii) the meta-analysis was limited to literature in Chinese and English, with the studies in Chinese accounting for a larger proportion, which is a potential source of bias. 


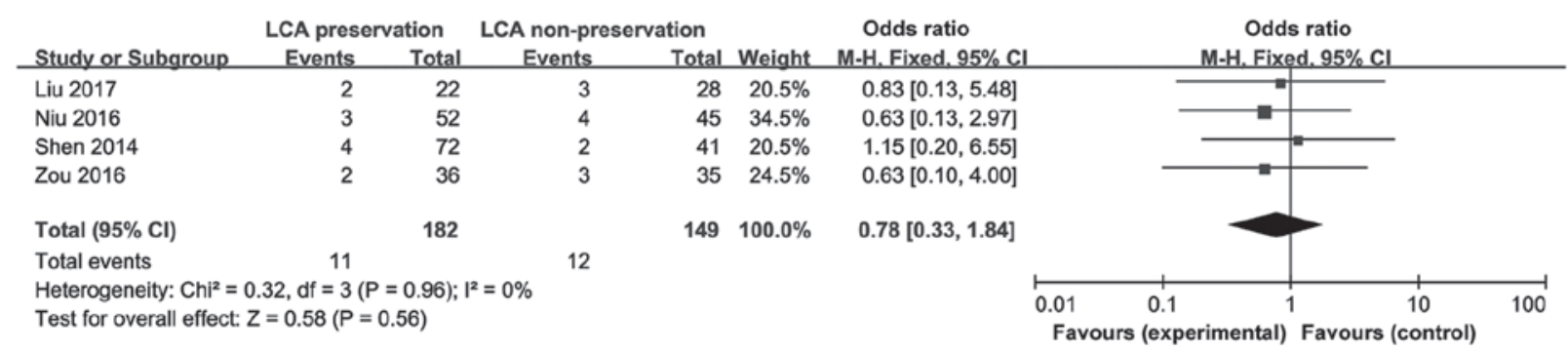

Figure 10. Meta-analysis of metastasis between the two groups.
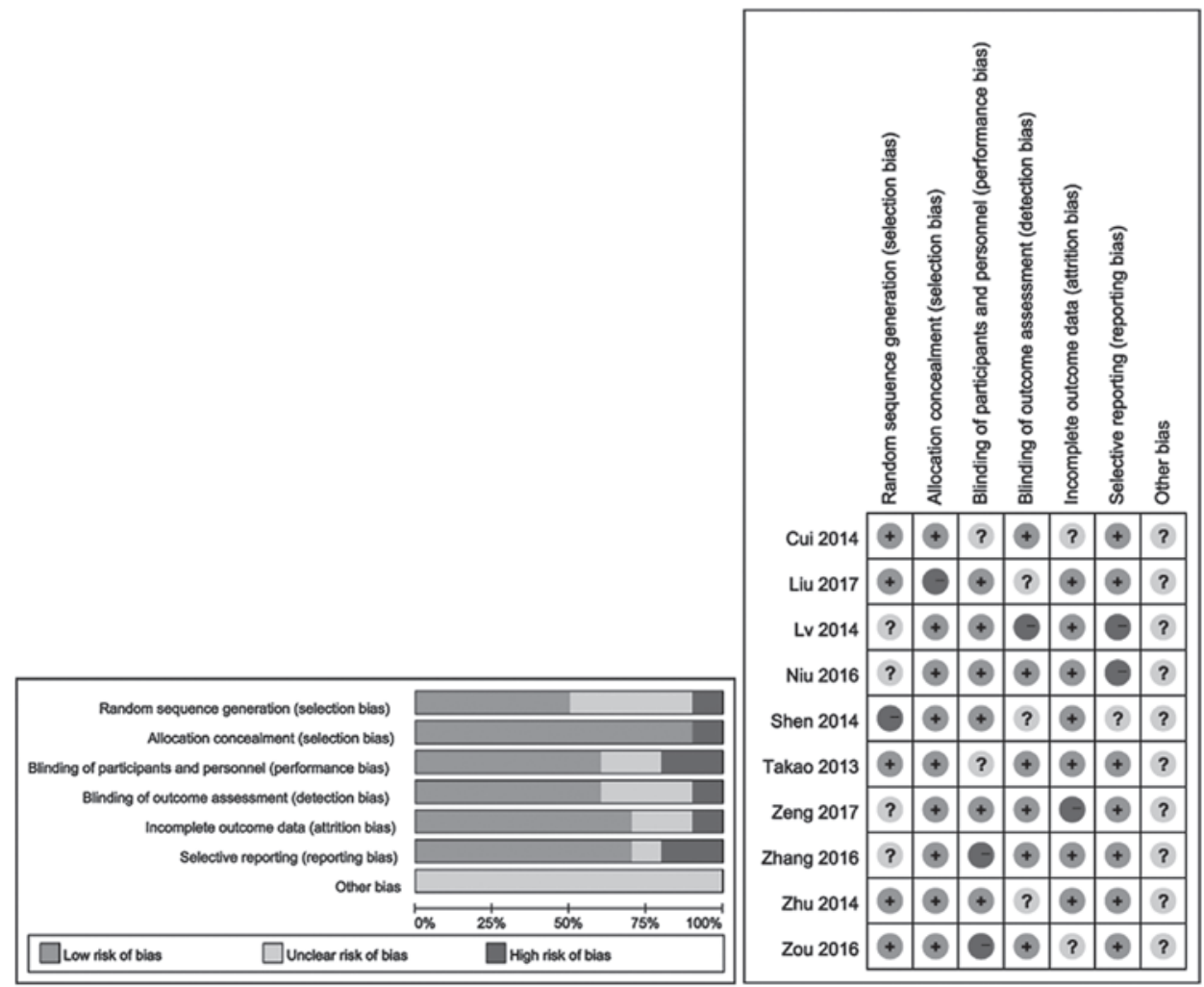

Figure 11. Quality evaluation of Cochrane risk of bias assessment.

In summary, the evidence presented herein suggests that preserving the LCA is associated with a more favourable outcome in laparoscopic resection for rectal cancer compared with non-preservation. Ensuring clinical efficacy requires long-term follow-up and further investigation. Due to the number and quality of research restrictions, the abovementioned conclusions require verification by further research with larger samples and high-quality RCTs.

\section{Acknowledgements}

Not applicable.

\section{Funding}

The present study was supported by the Graduate Innovation Program of Bengbu Medical College (grant no. Byycx1752).

\section{Availability of data and materials}

All data generated or analysed during this study are included in this published article.

\section{Authors' contributions}

Conceptualization: TJY, CSZ, DWF, XHL and CYY. Data curation: DWF, CSZ and TJY. Formal analysis: DWF, CSZ, XHL and CYY. Funding acquisition: TJY. Investigation: DWF, CSZ and XHL. Methodology: TJY, DWF and CSZ. Project administration: TJY and CYY. Resources: TJY, DWF. Software: XHL, DWF and CYY. Supervision: TJY. Validation: DWF, CSZ and XHL. Visualization: TJY, DWF and CSZ. Writing-original draft: DWF, CSZ and TJY. Writing-review \& editing: TJY.

\section{Ethics approval and consent to participate}

Not applicable. 


\section{Patient consent for publication}

Not applicable.

\section{Competing interests}

All the authors declare that they have no competing interests to disclose.

\section{References}

1. Zhang H, Yuan W, Zhou Q, Gu X and Wang F: Efficacy comparison of robotic and laparoscopic radical surgery in the treatment of middle-low rectal cancer. Zhonghua Wei Chang Wai Ke Za Zhi 20: 540-544, 2017 (In Chinese).

2. Bjørn MX and Perdawood SK: Surgical treatment of mid- and low rectal cancer. Ugeskr Laeger 179: pii: V11160801, 2017 (In Danish).

3. Kube R, Ptok H, Steinert R, Sahm M, Schmidt U, Gastinger I and Lippert $\mathrm{H}$ : Clinical value of laparoscopic surgery for colon cancer. Chirurg 79: 1145-1150, 2008 (In German).

4. Mabardy A, Lee L, Valpato AP and Atallah S: Transanal total mesorectal excision with intersphincteric resection and use of fluorescent angiography and a lighted urethral stent for distal rectal cancer. Tech Coloproctol 21: 581-582, 2017.

5. Guo Y, Wang D, He L, Zhang Y, Zhao S, Zhang L, Sun X and Suo J: Marginal artery stump pressure in left colic artery-preserving rectal cancer surgery: A clinical trial. ANZ J Surg 87: 576-581, 2015.

6. Niu JW, Ning W, Wang WY, Pei DP, Meng FQ, Liu ZZ and Cai DG: Clinical effect of preservation of the left colonic artery in laparoscopic anterior resection for rectal cancer. Zhonghua Yi Xue Za Zhi 96: 3582-3585, 2016 (In Chinese).

7. Zhang L, Zang L, Ma J, Dong F, He Z and Zheng M: Preservation of left colic artery in laparoscopic radical operation for recta cancer. Zhonghua Wei Chang Wai Ke Za Zhi 19: 886-891, 2016 (In Chinese).

8. Rutegård M, Hassmén N, Hemmingsson $\mathrm{O}$, Haapamäki MM, Matthiessen $\mathrm{P}$ and Rutegård $\mathrm{J}$ : Anterior resection for rectal cancer and visceral blood flow: An explorative study. Scand J Surg 105: 78-83, 2016.

9. Yasuda K, Kawai K, Ishihara S, Murono K, Otani K, Nishikawa T, Tanaka T, Kiyomatsu T, Hata K, Nozawa H, et al: Level of arterial ligation in sigmoid colon and rectal cancer surgery. World J Surg Onco 14: 99, 2016.

10. Yamamoto M, Okuda J, Tanaka K, Ishii M, Hamamoto $\mathrm{H}$ and Uchiyama K: Oncological impact of laparoscopic lymphadenectomy with preservation of the left colic artery for advanced sigmoid and rectosigmoid colon cancer. Dig Surg 31: 452-458, 2014.

11. Matsuda K, Hotta T, Takifuji K, Yokoyama S, Oku Y, Watanabe T, Mitani Y, Ieda J, Mizumoto Y and Yamaue H: Randomized clinical trial of defaecatory function after anterior resection for rectal cancer with high versus low ligation of the inferior mesenteric artery. Br J Surg 102: 501-508, 2015.
12. Tanaka J, Nishikawa T, Tanaka T, Kiyomatsu T, Hata K, Kawai K, Kazama S, Nozawa H, Yamaguchi H, Ishihara S, et al: Analysis of anastomotic leakage after rectal surgery: A case-control study. Ann Med Surg (Lond) 4: 183-186, 2015.

13. Liu F and Zhang LA: A clinical comparative study on reservation of left colic artery in laparoscopic resection of rectal cancer. J Clin Experimental Med 16: 1013-1016, 2017.

14. Shen J, Li MZ, Du YF, Qu H and Zhang Y: A comparative study of laparoscopic anterior resection of rectal carcinoma with and without preservation of the left colonic artery. Chin J Min Inv Surg 14: 22-24, 2014 (In Chinese).

15. Hinoi T, Okajima M, Shimomura M, Egi H, Ohdan H, Konishi F, Sugihara K and Watanabe M: Effect of left colonic artery preservation on anastomotic leakage in laparoscopic anterior resection for middle and low rectal cancer. World J Surg 37: 2935-2943, 2013.

16. Nonaka S, Saito Y, Takisawa H, Kim Y, Kikuchi T and Oda I: Safety of carbon dioxide insufflation for upper gastrointestinal tract endoscopic treatment of patients under deep sedation. Surg Endosc 24: 1638-1645, 2010.

17. Wolf FM: Meta-Analysis: Quantitative methods for research synthesis by Fredric M. 1st edition. Sage Publications Beverly Hills, CA, pp77-92, 1986.

18. Gu H, Wang Y and Li W: The application of cochrane risk of bias tools in meta-analysis of RCT. Chin Circ J 29: 147-148, 2014.

19. Murray AC, Chiuzan C and Kiran RP: Risk of anastomotic leak after laparoscopic versus open colectomy. Surg Endosc 30: 5275-5282, 2016

20. Leake PA, Plummer JM, Rhoden A, Frankson MA, Gordon-Strachan G, Powell LP and Roberts PO: Colorectal anastomotic leakage at the university hospital of the west indies: An analysis of risk factors. West Indian Med J 62: 711-715, 2013.

21. Tocchi A, Mazzoni G, Fornasari V, Miccini M, Daddi G and Tagliacozzo S: Preservation of the inferior mesenteric artery in colorectal resection for complicated diverticular disease. Am J Surg 182: 162-167, 2001.

22. Thomas MS and Margolin DA: Management of colorectal anastomotic leak. Clin Colon Rectal Surg 29: 138-144, 2016.

23. Sekimoto M, Takemasa I, Mizushima T, Ikeda M, Yamamoto H, Doki Y and Mori M: Laparoscopic lymph node dissection around the inferior mesenteric artery with preservation of the left colic artery. Surg Endosc 25: 861-866, 2011.

24. Dworkin MJ and Allen-Mersh TG: Effect of inferior mesenteric artery ligation on blood flow in the marginal artery-dependent sigmoid colon. J Am Coll Surg 183: 357-360, 1996.

25. Seike K, Koda K, Saito N, Oda K, Kosugi C, Shimizu K and Miyazaki M: Laser Doppler assessment of the influence of division at the root of the inferior mesenteric artery on anastomotic blood flow in rectosigmoid cancer surgery. Int J Colorectal Dis 22: 689-697, 2007.

26. Komen N, Slieker J, de Kort P, de Wilt JH, van der Harst E, Coene PP, Gosselink MP, Tetteroo G, de Graaf E, van Beek $\mathrm{T}$, et al: High tie versus low tie in rectal surgery: Comparison of anastomotic perfusion. Int J Colorectal Dis 26: 1075-1083, 2011 